\title{
Econophysics vs Cardiophysics: the Dual Face of Multifractality
}

\author{
Zbigniew R. Struzik \\ Centrum voor Wiskunde en Informatica (CWI) \\ Postbus 94079, NL-1090 GB, Amsterdam, The Netherlands \\ email: Zbigniew.Struzik@cwi.nl
}

Summary. Multifractality in physiological time series and notably in human adult heart rate has been primarily attributed to the Fourier phase ordering of the signal [1]. In contrast, the primary cause for the width of the multifractal spectrum in financial time series has recently been connected with fat-tailed distribution rather than phase ordering [2]. Reducing the source of multifractality of the human heart to nonlinear phase ordering may in fact not be entirely exhaustive or even correct. In the ultimate stress of the heart of the fetus during labour, there is an essential contribution of fat tails of the event distribution. Generally the multifractal spectra of the fetal heart rate during labour are considerably wider than the spectra for adults. We attribute the difference to the large shocks which have previously been shown to exhibit similar behaviour to financial index crashes [3].

Key words: Econophysics, Cardiophysics, Multifractal spectra, Non-linearity

\section{Introduction - econophysics versus cardiophysics?}

At first glance, there seems to be very little linking human heart rate and the stock index level, unless of course one is a trader or has invested a considerable amount of money... There may, however, be a much closer and physically and fundamentally more meaningful link than meets the eye between heartbeat neuro-regulation and market self-regulation.

There are a number of reasons why one may want to have a simultaneous look at the cardiac and economic systems. Both exhibit complex behaviour classified as scale invariant and multifractal $[1,4,5,6,7]$. Both can be seen as systems of a number of coupled oscillators, subject to stochastic regulation. Both consist of antagonistic actors; in the neuro-regulatory cardiac system, in addition to the pacemaker, the periodic pulse generating node, heart rhythm is driven by sympathetic and parasympathetic nodes, in constant imbalance. 
The sympathetic system slows down the heart rate, while the parasympathetic one accelerates it. One might think that during activity, only the parasympathetic system would be active, while during rest or sleep only the sympathetic one would be in action. Contrary to this, both systems are at work at any moment in time. In fact, it is a constant, simultaneous 'struggle' of both systems which gives rise to the actual heartbeat ratio, characterised by continuously changing dynamics $[1,8,9,10]$.

This scenario resembles a view of the market with pessimists who sell and optimists who buy stock options at any given instant. The number of actors changes as the market changes. Some pessimists join the optimist group and vice versa. Additionally, there is leakage of information, or simply similar action, clustering the behaviour of agents in both the pessimistic and the optimistic groups. This leads to a continuously varying number of active agents in the system, which may be related to the number of effectively active degrees of freedom of the abstract market system under consideration.

In particular, in the market scenario, polarisation of opinions leads to clusters of agents, effectively reducing the number of independent degrees of freedom in the system [11]. The resultant behaviour of the stock becomes (anti-)correlated and bends towards anti-persistent fractional Brownian motion characterised by $H<0.5$.

The behaviour of the healthy heartbeat is dominated by the coherent, concerted action of the inputs within the nodes of the two antagonistic systems (sympathetic and parasympathetic), leading to an extremely high level of anti-persistence at the range of $H=0.0$ to $H=0.1$ (for the normal healthy heart rate at rest). In the case of pathology, exercise or stress, this behaviour becomes less anti-persistent and less (anti-)correlated, potentially indicating less coherence in the functioning of the two antagonistic systems and thus possibly the increase of the effective independent degrees of freedom in the system.

The similarities drawn between both systems of market self-regulation and heart neuro-regulation can be expanded still further. In the condition of extreme stress during labour, the heart rate of the child being born can be shown to undergo a dynamical evolution which can resemble, and be compared to, severe stock market crashes [3].

In this article, we further elaborate on the reasons for comparing the neuroanatomic system of heartbeat regulation with the market. In particular we confront the view suggesting different origins of multifractality in financial time series [2] and physiological time series [1], by demonstrating that both mechanisms coexist in fetal heartbeat during labour. 

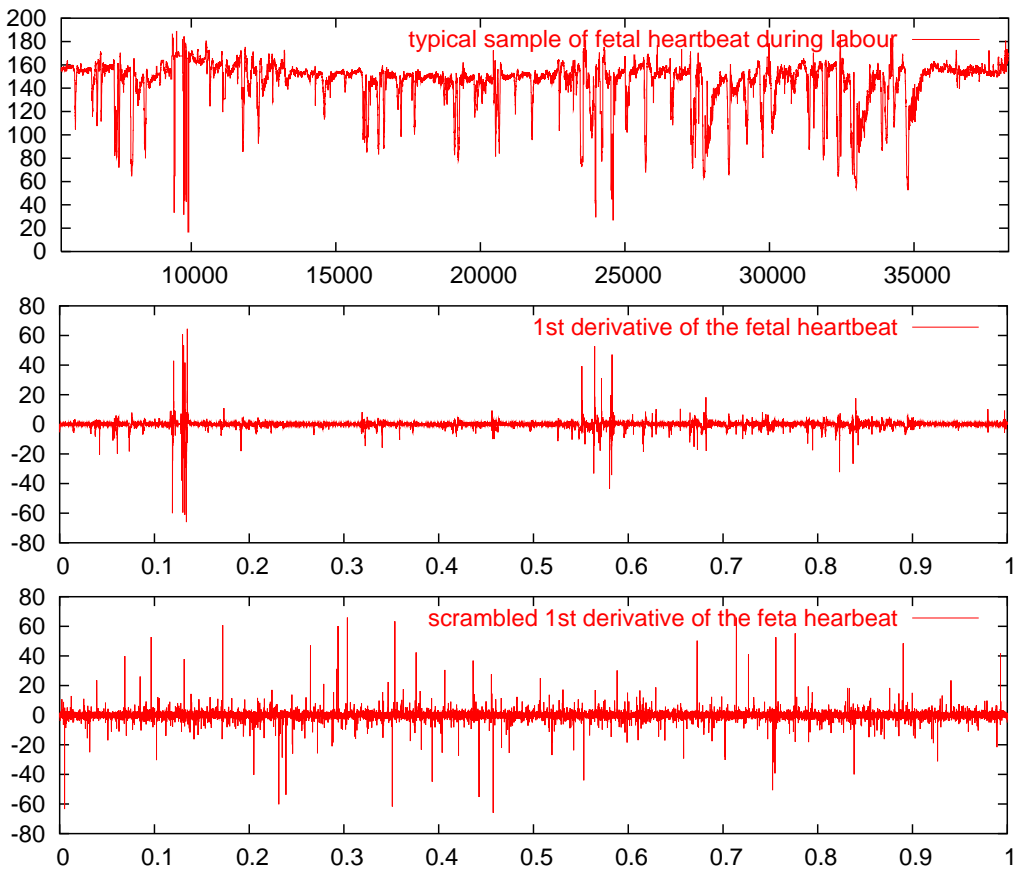

Fig. 1. Top: the input time series: fetal heart rate record. Middle: first derivative of the input time series. Bottom: scrambled first derivative of the input time series.

\section{The dual face of multifractality in fetal heartbeat}

Multifractality in physiological signals and notably in human adult heart rate has been primarily attributed to the Fourier phase ordering of the signal [1]. In contrast, the primary cause of the width of the multifractal spectrum in financial time series has recently been connected with the fat-tailed distribution rather than phase ordering [2]. In the same ref. [2], the distinction has been made between commodities and stock indexes, indicating that the commodities possess increased level of higher order temporal correlations which results in a wider multifractal spectrum than the stock indexes.

Reducing the source of multifractality of the human heart to nonlinear phase ordering only may, in fact, not be entirely exhaustive or even correct. In the ultimate stress of the heart of the fetus during labour, there is an essential contribution of extreme events resulting in fat tails of the event distribution. Generally the multifractal spectra of fetal heart rate during labour are considerably wider than the spectra for adults. We attribute the difference to the large shocks which have previously been shown to exhibit similar behaviour to the financial index crashes [3]. 
In figure 1 we show typical fetal heart rate during labour, followed by the first derivative of the signal which represents the 'returns' in the middle plot. Next, in the bottom plot of the same figure we show a scrambled version of the 'returns'. For scrambling we used random pairwise replacement of the returns carried out 1000 times the length of the time series, i.e. $10^{3} 2^{15}$ pair replacements. The multifractal spectra have been evaluated using the Wavelet Transform Modulus Maxima (WTMM) method. In figure 2 we show spectra for both the raw time series, for the first derivative - the 'returns' and for the scrambled 'returns'. For comparison, the same procedure has been applied to a random walk sample (the returns of the random walk are white noise).

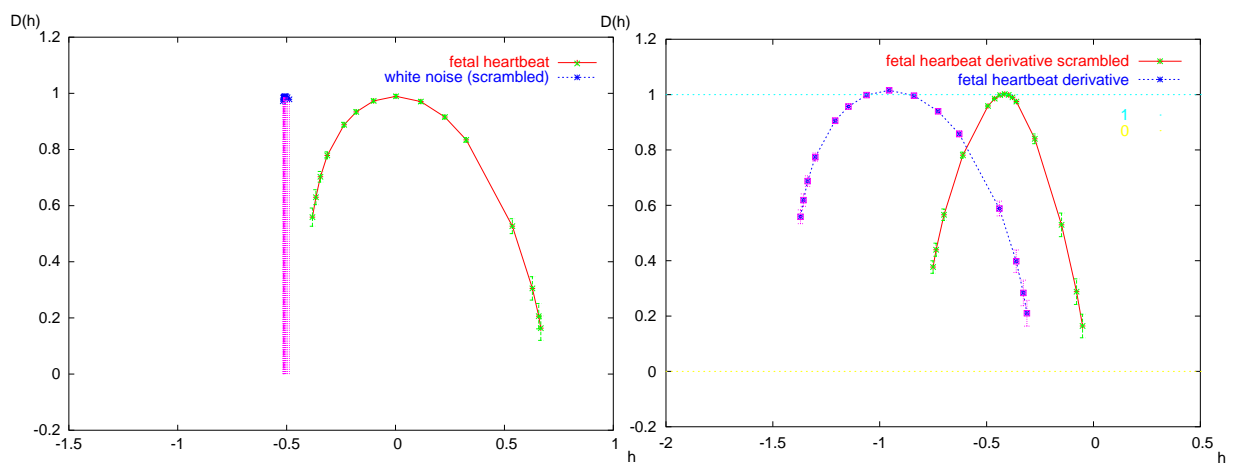

Fig. 2. Left: the MF spectrum of the raw fetal heart rate record and raw white noise. Right: the MF spectrum of the first derivative of the fetal heart rate record and its scrambled version.

As expected, there is no difference in the monofractal behaviour of the white noise. The difference in the behaviour of the multifractal spectrum of the fetal heart rate 'returns' suggests that the phase ordering substantially contributes to the width of the spectrum, as is the case with the healthy adult heart rate. Shown in figure 2 right, there is, however, a substantial nonvanishing residue, which we associate with the long tails in the distribution of the 'returns'. This behaviour resembles the duality of the multifractal properties in heart rate, in analogy to what has recently been discovered in financial time series (in particular of commodities) [2].

The presence of fat tails and their power law-like scaling is evident in the pdf shown in figure 3. Both the pdf of the fetal heart rate derivative and of a white noise record have been shown in linear-log and log-log coordinates. For the log-log plot, the modulus of the time series have been used and for the fetal heart rate it shows exponent 0.3 characterising the fat tails of the pdf. For comparison with finacial time series we plotted the pdf together with 

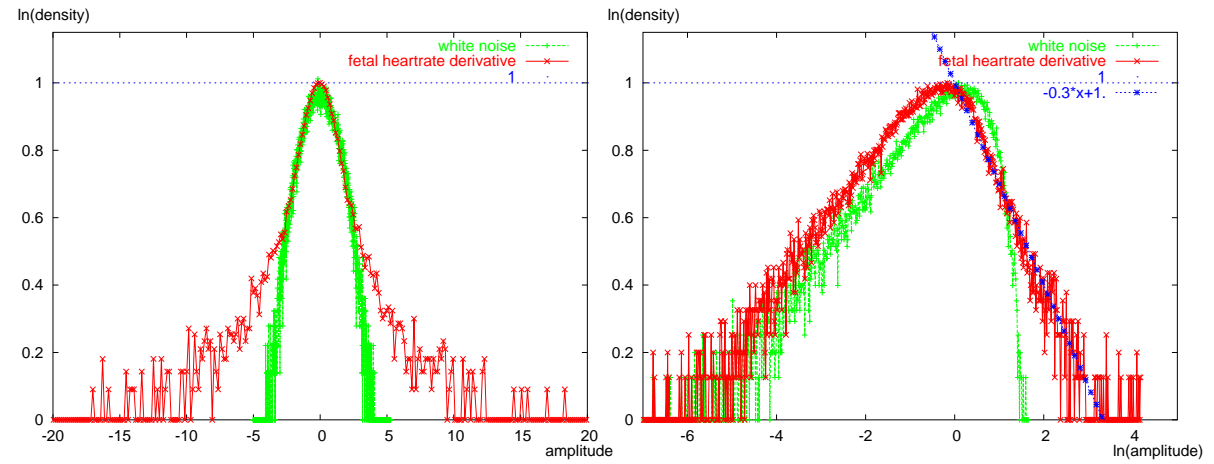

Fig. 3. Left: the fetal heart rate derivative shows fat tails in linear-log coordinates as compared to white noise. Right: the same in log-log coordinates; power law scaling with exponent 0.3 of the fat tails (positive and negative summed up) is evident.

the pdf of the returns of the S\&P500 index over the period of 1983-1991. The difference in power law decay of the fat tails is relatively small: in the samples compared the power law exponent for the fetal heart rate derivative is slightly smaller than that for S\&P500. This indicates even slightly higher contribution of the fat tails to the distribution, and in turn to the $D(h)$ multifractal spectrum. Additionally we tested the pdf shape for the fractionally differentiated fetal heart rate record, which brings it to the Hurst regime compatible with the stock index returns $H \approx-0.5+1$. Shown in figure 4 , we observe the formation of two regimes of scaling in the corresponding fetal heart rate pdf. This double regime is analogous to the double scaling regime of the pdf of S\&P500 returns [12].
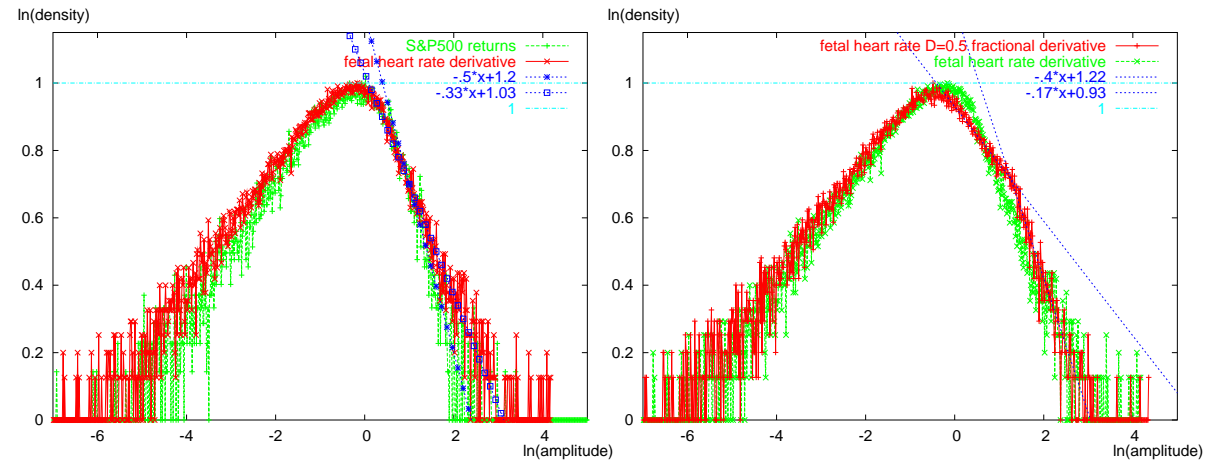

Fig. 4. Left: the fetal heart rate derivative fat tails in log-log coordinates scale with slightly lower exponent than S\&P500 record, indicating stronger tail presence. Right: fractionally differentiated fetal heart rate centred at $H \approx-0.5+1$ compatible with financial time series shows clear double scaling regimes. 


\section{Conclusions}

We have elaborated on similarities between heart rate and financial time series. The contribution of extreme events to the event distribution and multifractal spectra has been evaluated. We have confronted the view suggesting different origins of multifractality in financial time series and physiological time series by demonstrating that both mechanisms can coexist in heartbeat regulation in particular in fetal heartbeat during labour.

\section{Acknowledgments}

Discussions with Yosef Ahskhenazy and Takeshi Matsumoto are gratefully acknowledged.

\section{References}

1. P.Ch. Ivanov, M.G. Rosenblum, L.A. Nunes Amaral, Z.R. Struzik, S. Havlin, A.L. Goldberger and H.E. Stanley, Multifractality in Human Heartbeat Dynamics, Nature 399, 461-465, (1999).

2. K. Matia, Y. Ashkenazy, H.E. Stanley, Multifractal Properties of Price Fluctuations of Stocks and Commodities, Europhys. Lett., 61, (3), pp. 422-428 (2003).

3. Z.R. Struzik, Econonatology: The Physics of the Economy in Labour, Physica A, in press, (2003).

4. A. Fisher, L. Calvet, B.B. Mandelbrot, Multifractality of the Deutschmark/US Dollar Exchange Rate, Cowles Foundation Discussion Paper, (1997).

5. M.E. Brachet, E. Taflin, J.M. Tchéou, Scaling Transformations and Probablity Distributions for Financial Time Series, preprint cond-mat/9905169, (1999).

6. F. Schmitt, D. Schwertzer, S. Levejoy, Multifractal Analysis of Foreign Exchange Data, Appl. Stochasic Models Data Anal. 15, pp 29-53, (1999)

7. Y. Fujiwara, H. Fuijsaka, Coarse-graining and Self-similarity of Price Fluctuations, Physica A, 294 (3-4) pp.439-446, (2001).

8. M. Kobayashi, T. Musha, 1/f Fluctuation of Heartbeat Period, IEEE Trans Biomed. Eng., 29, 456-457 (1981).

9. C.-K. Peng, J. Mietus, J.M. Hausdorff, S. Havlin, H.E. Stanley, A.L. Goldberger Long-Range Anticorrelations and Non-Gaussian Bahavior of the Heartbeat Phys. Rev. Lett., 70, 1343-1346 (1993).

10. J.B. Bassingthwaighte, L.S. Liebovitch, B.J. West. Fractal Physiology, (Oxford University Press, 1994).

11. M. Zaluska-Kotur, S. Krukowski, A. Orlowski, Niestandardowa Analiza Fluktuacji Kursu Dolara Amerykańskiego, Proceedings of the 1st Conference on Modelling of the Economical Processes, 2002, Kielce, Poland (in Polish).

12. P. Gopikrishnan, V. Plerou,L.A. Nunes Amaral, M. Meyer, H.E. Stanley, Scaling of the Distributions of Fluctuations of Finacial Market Indices. Physical Review E, 60 (5), pp. 5305-5316, (1999). 